\title{
A STUDY OF MATERIAL RECOGNITION SYSTEM WITH HUMAN TACTILE SENSIBILITY FUNCTION USING PNEUMATIC CYLINDER
}

\author{
Chihiro HAGIWARA*, Osamu OYAMA* and Toshihiro YOSHIMITSU** \\ * Department of Mechanical Engineering, Faculty of Science and Technology \\ Meiji University Graduate School \\ 1-1-1 Higasimita, Tama, Kawasaki, Kanagawa, 214-0033 Japan \\ (E-mail: ce82045@isc.meiji.ac.jp) \\ ** Department of Robotics and Mechatronics, Faculty of Creative Engineering \\ Kanagawa Institute of Technology \\ 1030 Simoogino, Atsugi, Kanagawa, 243-0292 Japan
}

\begin{abstract}
We have developed an artificial finger which have human tactile sensibility function, such as be desired for some kind of robots. This finger can recognizes some materials pressing and tracing object with the arm actuated by the pneumatic cylinder. There are many factors in recognizing what touched material was. The recognition of materials is run by the neural network system which can be usefully to obscure and complicated control. The sensor in the finger has been used a piezo-electric element and a strain gauge with a thermo-couple respectively before. However only one of these sensors can be installed for one finger and the two or more fingers must be used to recognize one object. Then, the complete recognition is difficult for the reduction of measurement accuracy caused by individual try of many finger and the problem of the setting of output data in the neural network system. Therefore, the purpose in this report is to unify two or more sensors into one finger, and to fulfill the human tactile sensibility function more similar to human than previous subject. In this report, the improvement of the sensor and the device of the neural network are shown.
\end{abstract}

\section{KEY WORDS}

Artificial finger, Material recognition, Neural network system, Action potential

\section{INTRODUCTION}

The sense of touch information is necessary when we perform delicate operations such as grasp of objects. The interest rises to welfare and nursing in aging society in recent years, and it is welcome that robots work in such field. In this case, the robot fingers are required to have advanced function equal as human's one.

In this report, the artificial finger touches some materials actuated by pneumatic cylinder, and classifies the information from some sensors into 3 categories.
The first is "hardness" related hardness of surface. The second is "state on surface" related bump of surface. The third is "temperature" related thermal change of surface when the finger touched.

These 3 parameters are used for neural network system.

\section{DEVICE OF EXPERIMENT}

The recognition system is shown by Figure 1 . The main body is measurement part and is the artificial finger which is moved to axis of $\mathrm{x}$ and $\mathrm{z}$ using 2 pneumatic 
cylinders. The finger implants 4 strain gauges and a thermo-couple inside the imitating skin. The strain gauge is used for measurement of "hardness" and "state on surface" and the thermo-couple is used for measurement of "temperature". "State on surface" can't be obtained directly and then the differentiated value of the signal is used.

The values measured with the sensors are transmitted to the personal computer through the data logger. The datas are converted into the binary data imitating the impulse that simulates the excitement of human nerve. The system recognizes materials through the neural network at the end.

The measureing process is shown below.

1. The target material is fixed to the position on $3 \mathrm{~mm}$ from an artificial finger and then finger comes in contact with the target.

2. The pneumatic cylinder pushes the finger during 5 seconds and then feeds the finger sliding along the target.

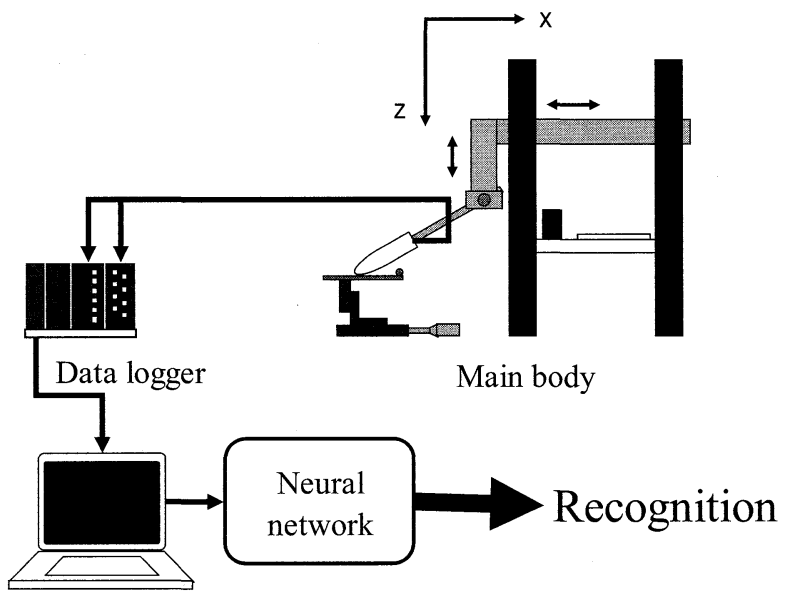

Figure 1 System of material recognition

\section{RESULT OF EXPERIMENT}

Figure 2 shows the system operations of learning the neural network based on measurement data. The cyclic calculation is shown on horizontal axis and the learning error is shown on vertical axis. The learning error means the average of square harmony of difference between true value and measurement value (LMS). This grants to know a degree of the learning.

In general, the LMS becomes 0.01 or less and then the system terminates to learn. In figure 2 , as the typical case, the LMS becomes 0.002651 in the cyclic calculation of 5000th times. Moreover, the neural network operates stably for unlearned data. As a result, the recognition rate, which shows the rate of recognized materials correctly, became $33.3 \%$, and the generalization error improved from 0.12 to 0.05 comparing former same condition. The reason of the improvement is mainly a change of teaching data for learning in neural network. The used data had been calculated by SD method since last year, but the proposed neural network uses the elements that respond particular inputs as the parameter, and are named each material name.

However, the correct recognition rate is not high as $33.3 \%$, the neural network sometimes causes not correctly recognition. This reason is that the measurement is not even for a different condition and a performance of the artificial finger is not sufficient. The improvement of hardware is needed for the more accurate recognition.

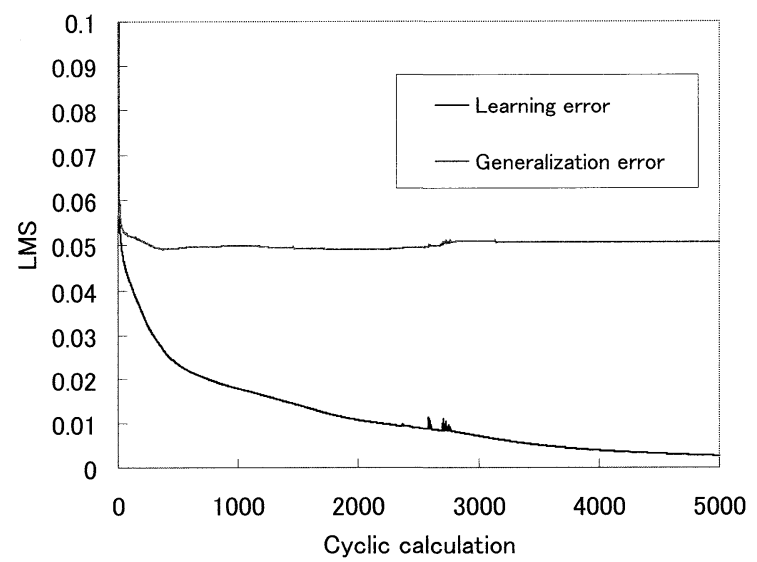

Figure 2 The learning error of neural network

\section{CONCLUSION}

We show that the proposed artificial finger is able to recognize some materials by using 3 parameters; "hardness", "state on surface", and "temperature". Moreover, this system deals the compressed binary data imitating the impulse of human nerve. This advantage is that the handling data of each sensor becomes lighter and the calculation becomes faster than ever. By this fact, it is expected that the artificial finger can have a lot of sensors. In addition, it shows to be able to substitute a low-cost microcomputer and FPGA in place of a high-cost personal computer.

The problem is uncertainty on measurement. It is need to improve a structure of the artificial finger, for example changing stuff and process of production.

The artificial finger will have a function similar to human to solve any problems. And the advanced robot hand will flourish in a care case in the future. 